\title{
Delayed onset isolated post-traumatic sixth nerve palsy due to trendelenburg position: an unusual cause
}

Thapa $A^{1}$, Shrestha $D^{2}$, Giri $S^{3}$

${ }^{1}$ Amit Thapa, Assistant Professor; ${ }^{2}$ Dibesh Shrestha, Medical Officer; ${ }^{3}$ Sandarva Giri, Medical Officer; Unit of Neurological Surgery, Department of Surgery, Kathmandu Medical College Teaching Hospital, Kathmandu, Nepal.

\section{Abstract}

We report here an unusual case of post-traumatic isolated sixth nerve palsy occurring while nursing the patient in trendelenburg position. This is the first case to be reported in the available literature of posture induced delayed onset isolated post-traumatic sixth nerve palsy and highlights importance of avoiding head down position while nursing patients with head injury.

Key words: Delayed, Head injury, Isolated traumatic sixth nerve palsy, Trendelenburg position

\section{INTRODUCTION}

$\mathrm{R}^{\mathrm{e}}$ everse Trendelenburg positioning of head injured patient has been prescribed in literature to decrease intracranial pressure (ICP). Sixth cranial nerve being the longest nerve is susceptible to rise in ICP. However there is no reference to posture induced delayed onset isolated post-traumatic sixth nerve palsy till date. We report this unusual clinical presentation.

\section{CASE REPORT}

A 34 year old woman while crossing road was hit by a motorbike. Soon following accident, she was escorted to Emergency Department of Kathmandu Medical College Teaching Hospital with history of transient loss of consciousness with two episodes of vomiting and had sustained fracture of shaft of left tibia. On examination her Glasgow coma scale (GCS) was 15/ 15 with no focal neurological deficit. Her left leg was splinted. Orthopaedic surgeons sought neurosurgical clearance for internal fixation of the tibial fracture. Her CT scan of the head revealed bifrontal basal contusion without any significant mass effect with right sided occipital lobe encephalomalacia. She was advised conservative care and orthopaedic intervention was put on hold. After 48

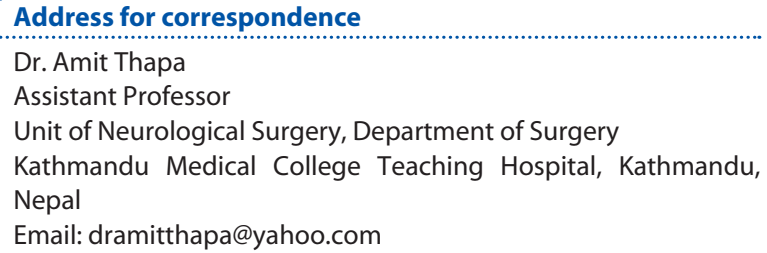

hours of injury she was advised lower limb traction by orthopaedic surgeon and to achieve counter traction her upper body was kept in Trendelenburg position. After an overnight traction she complained of diplopia while looking towards her right side. Examination revealed isolated right-sided lateral rectus palsy. Other cranial nerves as well as her higher mental functions were normal. Her repeat non-contrast CT scan of head did not show any increase in size of the intracranial hematoma or any mass effect. She was managed conservatively. After two weeks of injury her condition remained stable with slight improvement in her gaze towards her right side. Her tibial fracture was internally fixed.

\section{DISCUSSION}

The sixth cranial nerve due to its longest intracranial extraparenchymal subarachnoid course is prone to compression injury due to mass effect as well as traction injury. Located in the pons, just ventral to floor of the fourth ventricle and just lateral to the medial longitudinal fasciculus (MLF), sixth nerve nucleus innervates ipsilateral lateral rectus to cause abduction of ipsilateral eye. Patient with sixth nerve palsy presents with binocular horizontal diplopia (more while looking towards paretic eye) and esotropia in primary gaze.

Among the causes for isolated abducens nerve palsy, Berlit et al in a series of 165 patients found trauma in just $3.1 \%$ compared to vascular causes in $29.7 \%$, inflammatory diseases $19.4 \%$ and tumours $10.9 \%$. Non-traumatic isolated sixth cranial nerve paresis is commonly seen due to post viral syndrome in paediatric 
patients and ischaemic mononeuropathy in adults. Posttraumatic abducens nerve palsy is usually unilateral². Mechanical entrapment of extraocular lateral rectus muscle as seen in blow out fracture of the orbit should be ruled out before implicating traumatic abducens nerve paresis as previous condition demands immediate surgical intervention. Delayed onset (i.e. not present immediately after accident) post-traumatic abducens paresis has been reported to be due to chronic subdural haematoma or oedema/ischaemia due to injury to soft tissue structures housing these nerves ${ }^{3}$. However no case has been reported in the published literature due to posture induced sixth nerve paresis. In this patient, encephalomalacia located in occipital lobe noted on CT scan of head was too far located and too small to cause direct nerve compression. Beside this, no obvious intracranial mass lesion or features of cerebral herniation was found. Trendelenburg position that involves putting head lower than heart level is known to induce intracranial hypertension ${ }^{4}$. In absence of known causes of post traumatic sixth nerve paresis noted above, we hypothesize that during Trendelenburg position; due to cerebral swelling sixth nerve could have been compressed leading to its paresis.

Traumatic abducens nerve palsies are usually managed conservatively though Kao et al showed better recovery with subtenon botulinum toxin injection ${ }^{5}$. Mutyala et al have reported spontaneous recovery in $27 \%$ cases with unilateral traumatic abducens nerve palsy where as only $12 \%$ with bilateral palsy improve 6 . In our case, due to its reversible pathology, we managed her conservatively and she gradually improved over two weeks follow up. Initial conservative treatment is recommended before strabismus surgery for non-resolving sixth nerve paresis.

\section{CONCLUSION}

Trendelenburg position can cause delayed onset posttraumatic sixth nerve paresis in patients with head injury. This case highlights the importance of positioning in patients with head injury to avoid cerebral edema and is as such the first case to be reported due to trendelenburg position.

\section{REFERENCES}

1. Berlit P, Reinhardt-Eckstein J, Krause KH. Isolated abducens paralysis-a retrospective study of 165 patients. Fortschr Neurol Psychiatr. 1989;57:32-40.

2. Dwarakanath S, Ravichandra, Gopal S, Venkataramana NK. Post-traumatic bilateral abducens nerve palsy. Neurol India. 2006;54:221-2.

3. Salunke P, Savardekar A, Sura S. Delayed-onset bilateral abducens paresis after head trauma. Ind J Ophthalmol. 2012;60(2):149-50.

4. Kalmar AF, Foubert L, Hendrickx JF, Mottrie $A$, Absalom A, Mortier EP, et al. Influence

of steep Trendelenburg position and $\mathrm{CO}_{2}$ pneumoperitoneum on cardiovascular, cerebrovascular, and respiratory homeostasis during robotic prostatectomy. $\mathrm{Br} J$ Anaesth. 2010;104(4):433-9.

5. Kao LY, Chao AN. Subtenon injection of botulinum toxin for treatment of traumatic sixth nerve palsy. J Pediatr Ophthalmol Strabismus. 2003;40:27-30.

6. Mutyala S, Holmes JM, Hodge DO. Younge BR. Spontaneous recovery rate in traumatic sixth-nerve palsy. Am J Ophthalmol. 1996;122:898-9. 